\title{
Percutaneous biopsy of retroperitoneal lesions - 10 year experience of a single centre
}

\author{
Petr Dvorak ${ }^{\mathrm{a}}$, Petr Hoffmann ${ }^{\mathrm{a}}$, Michal Balik $^{\mathrm{b}}$, Martina Hoffmannova ${ }^{\mathrm{c}}$, Jindrich Kopecky ${ }^{\mathrm{d}}$, Radka Dvorakovaa ${ }^{\mathrm{a}}$, Marketa Nova ${ }^{\mathrm{e}}$
}

\begin{abstract}
Aims. To retrospectively evaluate the technical features, efficacy, accuracy, appropriate complications, and relationships among monitored parameters of computed tomography-guided biopsies of the retroperitoneum-located processes. Methods. From December 2008 to December 2018, 208 percutaneous biopsy procedures for tumors, sized 14 - 190 mm in diameter (median size $57.5 \mathrm{~mm}$ ), were performed on patients with suspected retroperitoneal tumorous process on imaging examinations. The patients were men in 124 cases and women in 84 cases, aged 20 to 90 years (median age 63.2 years). Skin to lesion distance was variable; from $43 \mathrm{~cm}$ to $178 \mathrm{~cm}$ (median length $108.5 \mathrm{~cm}$ ).

Results. In 202 cases (97.1\%) results were true positive or true negative; only 6 interventions (2.9\%) were histologically false negative and had to be confirmed surgically. DLBCL and metastatic disease to the retroperitoneal lymphatic nodes were the most common diagnoses ( $23.1 \%$ each); lymphoma types were verified in $40.9 \%$ of cases. 7 complications in total were revealed, 6 of which were minor hemorrhages, and in one case ureteral injury was detected. A statistically significant relationship between the hypervascular process and complication incidence $(P=0.00166)$ and needle gauge $(P=0.01427)$ was identified.

Conclusion. Percutaneous CT-guided biopsy performed in patients with a suspected retroperitoneal tumorous process had a high accuracy in establishing the correct diagnosis including histological subtyping. Simultaneously, the complication rate was low.
\end{abstract}

Key words: core needle biopsy, retroperitoneal process, percutaneous approach, complications, hypervascular mass.

Received: April 13, 2019; Accepted: June 3, 2019; Available online: June, 17, 2019

https://doi.org/10.5507/bp.2019.028

(c) 2020 The Authors; https://creativecommons.org/licenses/by/4.0/

${ }^{a}$ Department of Radiology, Charles University, Faculty of Medicine and University Hospital, Hradec Kralove, Sokolska 581, 500 05, Czech Republic

${ }^{b}$ Department of Urology, Charles University, Faculty of Medicine and University Hospital, Hradec Kralove, Sokolska 581, 500 05, Czech Republic

'Faculty of Education, University of Hradec Kralove, Hradec Kralove, Rokitanskeho 62, 500 03, Czech Republic

${ }^{d}$ Department of Oncology and Radiotherapy, Charles University, Faculty of Medicine and University Hospital, Hradec Kralove, Sokolska 581, 500 05, Czech Republic

'The Fingerland Department of Pathology, Charles University, Faculty of Medicine and University Hospital, Hradec Kralove, Czech Republic Corresponding author: Petr Hoffmann, e-mail: petr.hoffmann@fnhk.cz

\section{INTRODUCTION}

The retroperitoneal space except for the pancreas, duodenum, kidneys and adrenal glands constitutes the anatomically thin part of the abdominal cavity between the posterior parietal peritoneum and anterior to the transversalis fascia. In unaffected conditions, it contains connective tissue with the ureters, vessels, nervous, and lymphatic structures around the abdominal aorta and inferior vena cava with their branches ${ }^{1}$.

The spectrum of possible impairments is wide, the affections frequently show relatively unimpeded growth, where clinical symptoms develop late and the processes at presentation tend to be large. The average size exceeds 10 centimeters at the largest diameter ${ }^{2}$. Primary retroperitoneal tumors are both rare and a heterogenous group of variable biological potential. Tumors can be mesenchymal (fat, muscle, fibrous tissue, peripheral nerve or nerve sheath, and vessels), epithelial or of extragonadal germinal cell origin ${ }^{3}$. Secondary retroperitoneal affections are common. Metastatic disease of the abdominal organs to the lymphatic nodes is an integral part of TNM classification. Lymphomas are the most common retroperitoneal disease ${ }^{4}$. Many benign processes have tumor-like morphological features and manifestations. It is crucial to obtain a valid histological sample of these lesions for the establishment of correct diagnosis and planning of further therapies ${ }^{5}$.

Historically, establishing the diagnosis was associated with a surgical procedure using laparotomy or laparoscopy ${ }^{6}$. The advantage was enormously high diagnostic accuracy, on the other hand this approach is demanding for patients who also carry all risks connected to general anesthesia. The majority of cases (especially lymphomas and metastatic lymphatic nodes) are systemic diseases and the treatment is non-surgical. Minimally invasive diagnostic methods using different instruments and various image guidance are accepted as an effective and safe alternative to surgery. Since initial experience in the 1980's and their gradual introduction into clinical practise using 
increasingly more sophisticated device, simultaneously with histological examinations, the results are constantly improving. We want to present our long-term results from the last decade, verify the usefulness of the procedure and point out important features of the technique.

\section{MATERIALS AND METHODS}

From December 2008 to December 2018, a group of 208 patients from the population of the Czech Republic was retrospectively evaluated. Indication for the biopsy was based on imaging examinations; in the first step ultrasonography was usually used. However, in all cases the suspicion was verified by contrast medium enhanced computed tomography (CT) or 18F-fluoro-2-deoxyglucose (18F-FDG) labelled positron emission tomography/computed tomography (PET/CT).

Measured parameters were the size at the largest diameter, skin to lesion distance and mass vascularization. Other monitored parameters were age, gender, biopsy needle gauge, number of bioptic attempts, known different tumorous diseases in the patients' clinical history, complications and their solutions, final histological diagnosis including subtyping if necessary for indicated physician, performed surgical excision biopsy in parallel with percutanous approach, and correlations with results of selected therapy.

The biopsies were performed at various anatomical levels of the retroperitoneum. The patients' prone or side position on CT table was suitable in all cases; a supine position was never needed. All of these interventions were performed under CT guidance. The device Siemens Somatom Definition AS Plus (Siemens, Forchheim,
Germany) was used. CT fluoroscopy guidance was never used. All the patients were indicated by the multidisciplinary council (radiologist, hematologist/ hematooncologist or oncologist, urologist or surgeon).

Fully informed consent was obtained in all cases by the biopsy-performing physician with an explanation of the procedure course and principles, the resulting consequences, possible complications, and their eventual solutions. This study was approved by the institutional ethics review committee. The procedures were performed using the Core semi-automatic biopsy system (Palium biopsy, M.D.L. SRL, Delebio, Italy). In all cases, a one-step approach was used; the coaxial technique was never performed.

The proper procedure was planned according to a preprocedural CT or PET/CT imaging examination. The shortest and safest needle distance to reach the lesion was established. The needle track was planned to avoid vessels and expected nerves pathway. In five complicated cases, a transcaval approach to reach the tumorous process was used (Fig. 1). In two cases, the tumorous mass arose in the retroperitoneum around the inserted aortic stentgraft. The biopsy needle track was planned maximise closeness to vessel structures and as well as safety.

The entry point was defined by placing a mark on the skin. The distance and biopsy needle angle were measured on the basis of a local anesthesia needle short CT imaging. Skin was disinfected and covered with sterile drapes exposing only the entry site. In accordance with the predetermined route, a $14 \mathrm{G} / 16 \mathrm{G} / 18 \mathrm{G} / 20 \mathrm{G}$ of $13 \mathrm{~cm} / 16 \mathrm{~cm} / 20 \mathrm{~cm}$ length with a $22 \mathrm{~mm}$ throw needle was inserted in the proper position into the lesion. After obtaining the sample, the needle was carefully removed from the patient's body. The material was put into a sterile

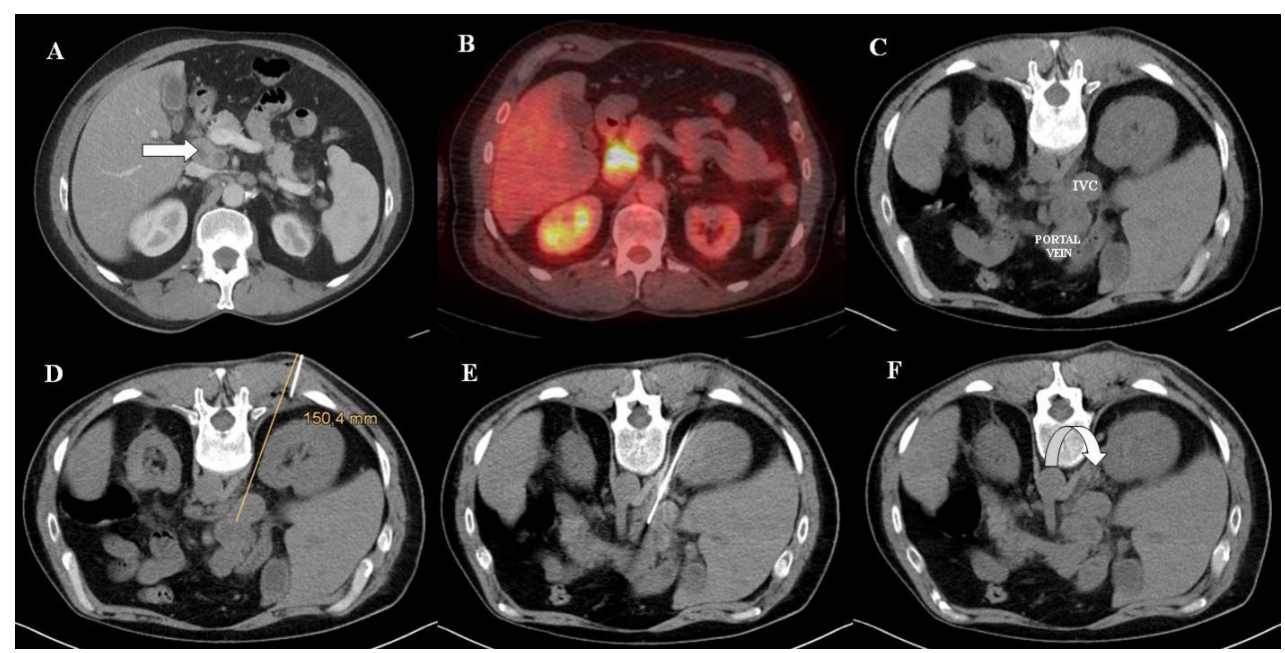

Fig. 1. The biopsy in the retroperitoneum using transcaval approach. Affected lymphatic nodes between large vessels in transversal plane (A, arrow) on contrast medium enhanced CT and simultaneously proven viable mass on PET/CT (B). Preprocedural CT examination confirms the lymphnode localization between inferior vena cava and portal vein, patient in prone position $(C)$. The placement of the local anesthesia needle and planning the track (D) and insertion of the biopsy needle (E, patient in prone position). The control CT examination revealed only minor perinodal and perivascular changes (F, curved arrow). The histological result was Hodgkin lymphoma, subtype nodular sclerosis. 


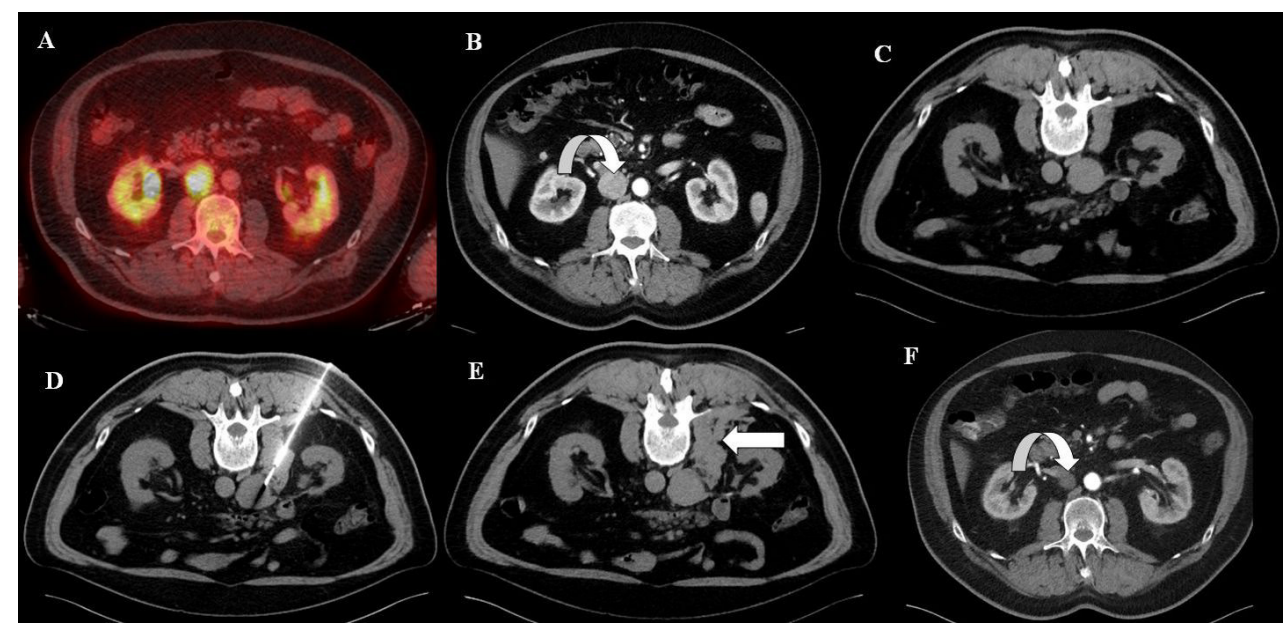

Fig. 2. The hemorrhage. Viable mass in retrocaval retroperitoneal space on FDG enhanced PET/ CT (A). The hypervascular mass on contrast medium enhanced CT in the transversal plane and arterial phase (B, curved arrow). Preprocedural CT examination confirms the localization near large vessels, patient in prone position $(C)$. The placement of the biopsy needle (D, patient in prone position), retroperitoneal hemorrhage was present straight after the procedure (E, arrow). The histological result was neuroendocrine carcinoma. Only residual avascular process was revealed by contrast medium enhanced CT examination after 4 months of targeted therapy (F, curved arrow).

36-38\% formaldehyde solution and placed into the transport system for histological examination.

All procedures were carried out using only local anesthesia (Trimecaine, Zentiva, Prague, Czech Republic); neither conscious sedation nor general anesthesia was never needed. Parameters of blood coagulation, International Normalized Ratio (INR, lower than 1.5) and activated Partial Thromboplastin Time (aPTT, lower than 1.5) were noted before the biopsy. After the intervention, one series of CT scans in the same ranges as in the preprocedural examination was performed to exclude the possibility of early complications; bleeding being the complication looked for first. The point of insertion into the skin was cleaned with a surgical scrub. The duration of the whole procedure, including all preparations, never exceeded 30 minutes. The patients were monitored for the remainder of the day by the standard department of hematology, oncology, urology or surgery. The next morning, they were discharged from the hospital after clinical, laboratory, and ultrasound examinations.

In total, 7 patients were not discharged from the hospital the next morning. In six cases, retroperitoneal hemorrhage was revealed straight after the procedure (Fig. 2). The patients' clinical status was stable, so a conservative approach using analgesia was sufficient in these cases. Blood transfusion was never necessary. These patients stayed in the hospital one day more, for a total of two days. In one difficult case, the biopsy was performed without immediate postprocedural complications. The patient got gradually worse, with increasing right lumbar pain. A CT examination was performed 6 days after the intervention, including delayed excretory phase, and an ureter injury was revealed (Fig. 3). The patient was then treated by insertion of an ureteral stent and stayed in the hospital for a total of 14 days; surgical revision was not necessary.
In surgically treated patients, histological results were correlated after the biopsy and after the operational approach. It was considered a success when the relationship between the histological results of the biopsy and the surgical resection were identical. In conservatively treated patients (lymphomas, disseminated malignancies and benign histological findings), biopsy results were correlated with the findings of the targeted treatment or known tumorous disease in their clinical history. Conservative treatments with a positive match were considered identical to findings of biopsy results and bone marrow evaluations in lymphoma cases, or to tumorous tissue amounts that appeared to be significantly decreasing or dissapearing on follow-up imaging examinations. If, despite the appropriate therapy, tumorous progression was revealed alongside patient fatality, the autopsy results were correlated with histological findings from the biopsy. For the follow-up, CT (equal CT device) or PET/CT Discovery VCT 64 (General Electric Healthcare, Milwaukee, Wisconsin, USA) was used. In some sporadically indicated situations (e.g. suspected retroperitoneal fibrosis or neurogenic origin affections), magnetic resonance was performed. Siemens Magnetom Avanto 1.5 Tesla (Siemens, Forchheim, Germany) was used.

If a negative histological result was obtained and a present tumorous process was still suspected, a surgical approach for obtaining tissue samples was used. Percutaneous rebiopsy was never performed. In 6 cases surgical excision biopsy was performed due to a negative percutaneous histological result, and in 12 cases despite a positive result. This course was used at the beginning of the study, when surgery was a gold diagnostic standard. In positive percutaneous cases were histological results identical; a combination of both techniques was used due to initial mistrust of a novel approach.

Histological results were accompanied with subtyping in indicated cases; the decisions for subtyping were made 


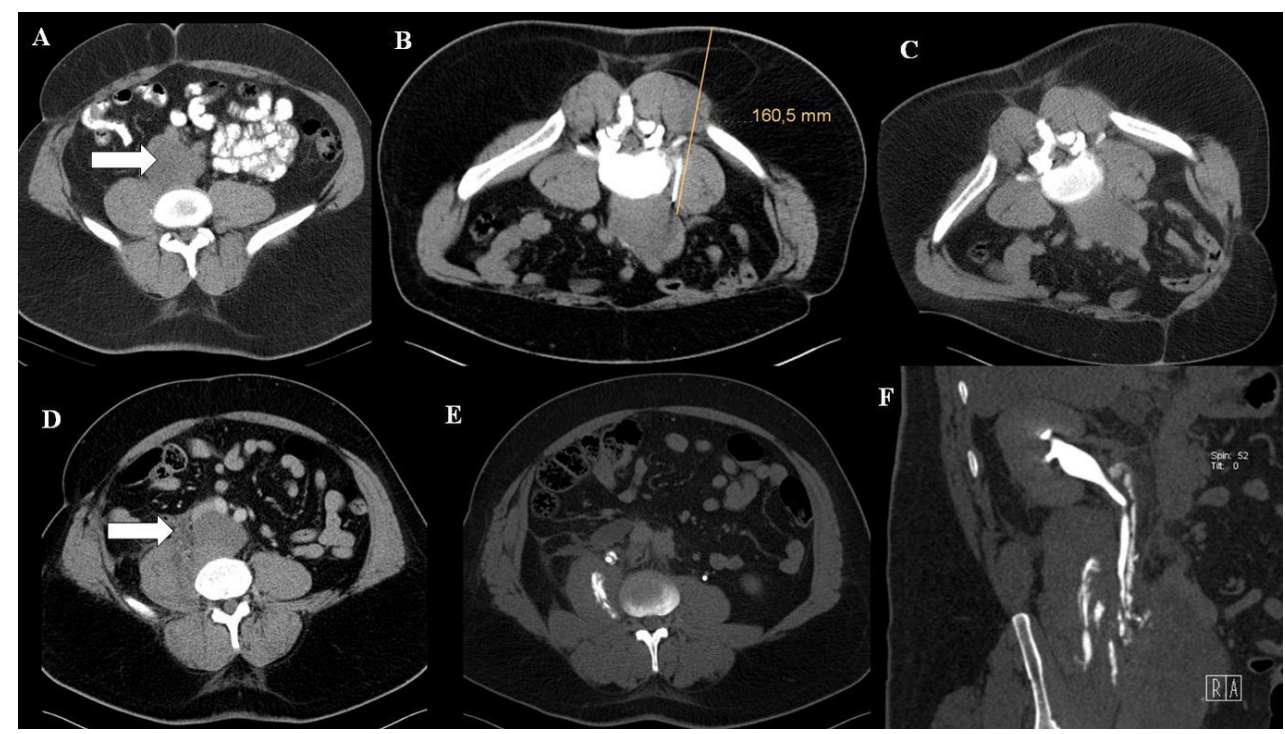

Fig. 3. The ureter injury. Hypovascular mass in lower levels of the retroperitoneum on contrast medium enhanced CT examination (A, arrow). Planning the track and inserting of the biopsy needle (B), patient in prone position. The control CT examination straight after the intervention revealed no complications (C). The histological result was ganglioneuroma. CT examination was performed 6 days after the intervention in the portal venous phase (D) and delayed excretory phase in the transversal plane (E, arrow) and oblique reconstruction $(\mathrm{F})$. The urine leakage proved the ureter injury.

by treating physicians. In verified cases of neuroendocrine tumors, nuclear grade 1 to 3 was established; in renal cell carcinoma, particular histological types (clear cell, papillary, chromophobe or rare) and also nuclear or nucleolar grading were determined, and in prostate cancer a Gleason score was necessary. In germinal cell neoplasms, individual tumorous parts were determined. In all cases of verified breast carcinoma, immunohistochemical examinations were performed due to occurrence of hormonal receptors; in colorectal carcinoma, genes RAS and BRAF mutations were required in sporadic cases. In diagnosed non-small cell lung cancer (NSCLC), histological subtype was also specified.

In Hodgkin disease, subtypes (nodular sclerosis, mixed cellularity, lymphocyte-rich or lymphocyte-depleted) were determined. In non-Hodgkin lymphoma diagnose, subtyping was absolutely crucial; detailed differentiation of indolent types - chronic lymphocytic leukemia/small lymphocytic lymphoma (CLL/SLL), marginal zone lymphoma or plasma cell lymphoma - and aggressive types - diffuse large B-cell lymphoma (DLBCL) or mantle cell lymphoma - determined the treatment as well as the prognosis. Examination of the follicular lymphoma was particularly a detailed category due to correct grading establishment especially in grades 2, $3 \mathrm{~A}$ and $3 \mathrm{~B}$.

For the study we used retrospective data collection. For the basic quantitative statistical evaluation, median and interval data was used. These parameters were correlated with number of bioptic attempts, needle gauge, skin to lesion distance, vascularization, surgical excision biopsy, histological accuracy including subtyping, and complications using Fisher's exact tests with contingency tables. Qualitative statistical data was descriptively evaluated and quantitative parameters were found. For evaluation of unpaired parameters, a nonparametric Mann-Whitney test was used. The statistical importance was established at level $P=0.05$. The statistical software NCSS 11 (NCSS, LLC, Kaysville, Utah, USA) was used.

\section{RESULTS}

A total of 208 interventional procedures with accessible histological results were included in the study. 208 biopsies in 208 patients were performed. The patients were men in 124 cases (59.6\%) and women in 84 cases ( $40.4 \%$ ), aged 20 to 90 years (median age 63.2 years). The percutaneous biopsies were performed in various retroperitoneal localizations for tumors ranging from 14-190 mm in diameter (median size $57.5 \mathrm{~mm}$ ). Overall, in only 6 interventions were histological results considered false negative $(2.9 \%)$. In 202 cases $(97.1 \%)$ results were considered true positive or true negative. A prone position was used in $181(87 \%)$ procedures and a side position in 27 (13\%) cases.

Histologically, the most common diagnoses were DLBCL and metastatic disease to the retroperitoneal lymphatic nodes, each occuring 48 cases (23.1\%). Other affections were revealed less frequently. None malignant cells were verified in a total of 28 interventions (13\%). A detailed overview can be found in Tables 1 and 2 .

In a total of 85 cases the diagnoses were lymphomas ( 85 of 208 cases; $40.9 \%$ ). DLBCL was verified as the initial diagnosis in 28 procedures, as relapse in 10 interventions, and as transformation of low grade follicular lymphoma in 3 patients and in 7 patients with CLL/SLL 


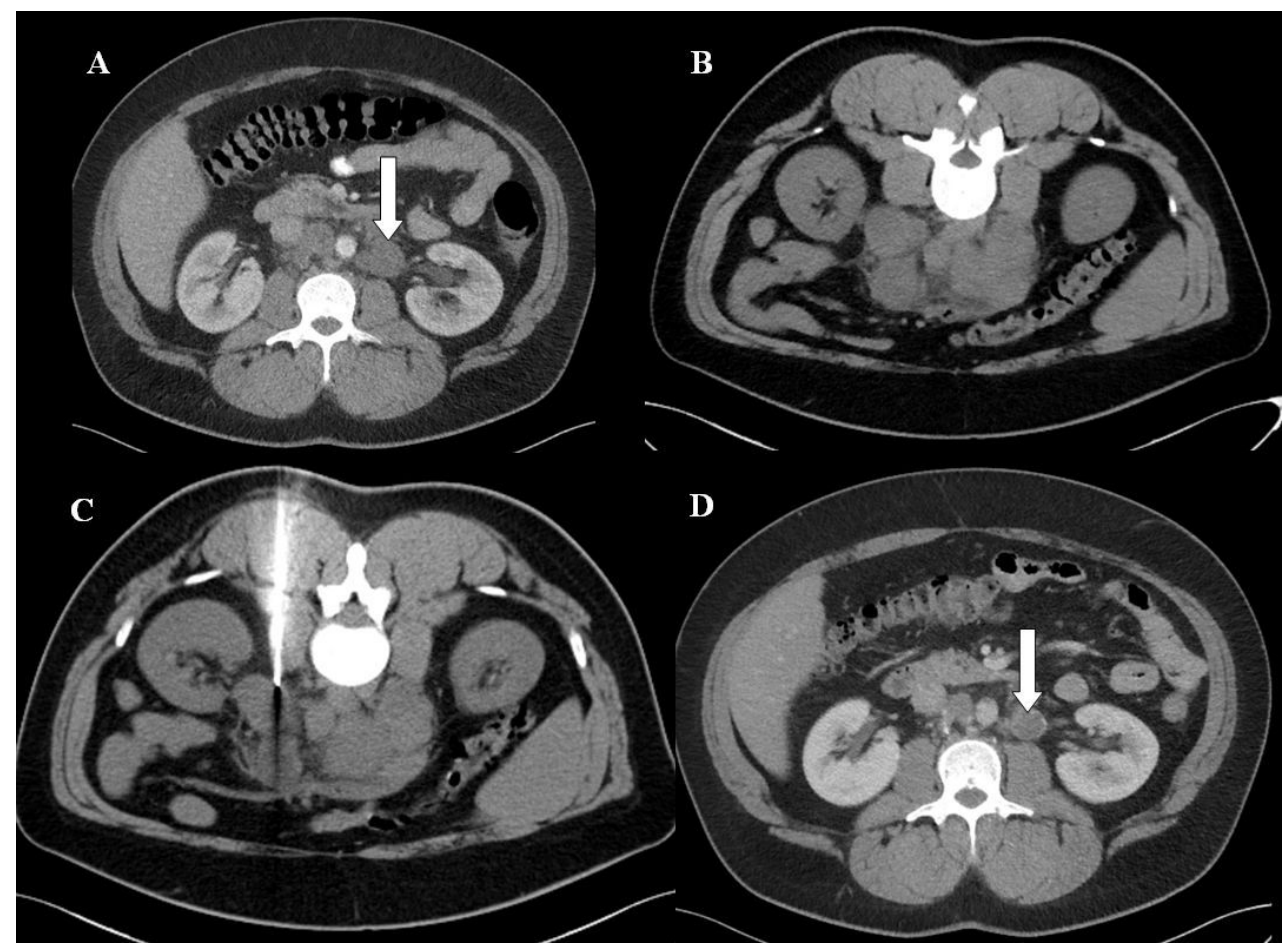

Fig. 4. The false negative result. Hypovascular lymph nodes in the left paraaortal region on contrast medium enhanced CT examination (A, arrow). Preprocedural CT examination (B, patient in prone position) and inserting of the biopsy needle $(\mathrm{C})$. The histological result was completely necrotic tissue. Surgical excision biopsy was performed and germinal cell tumor was verified. Targeted systemic treatment was indicated; follow-up CT examination 4 months after establishment of diagnosis was performed with partial regression verification $(\mathrm{D}$, arrow).

in clinical history, i.e. Richter's syndrome. Follicular lymphoma was revealed as the initial diagnosis in 15 cases and as relapse in 6 cases. CLL/SLL was established initially in 3 biopsies and as relapse in 7 patients. Other lymphoma subtypes were diagnosed rarely - mantle cell lymphoma 3 times and marginal zone lymphoma, plasma cell lymphoma and T-cell non-Hodgkin lymphoma one time each.

The $16 \mathrm{G}$ needle was utilized in 176 biopsies ( $84.6 \%$ ), the $18 \mathrm{G}$ needle in 27 interventions (13\%), the $14 \mathrm{G}$ needle in 3 cases $(1.4 \%)$ and $20 \mathrm{G}$ in 2 procedures $(1 \%)$. The $16 \mathrm{G}$ needle was sufficient for the majority of cases. The lower calibre needles ( $18 \mathrm{G}$ and $20 \mathrm{G}$ ) were used in risky affected areas located near great vessels and in cases where hypervascular process was preproceduraly diagnosed. The Fisher's exact test showed a statistically significant relationship between the needle gauge and the hypervascular process $(P=0.01427)$.

The number of bioptic attempts was variable. One sampling in 21 cases (10.1\%), two samplings in 141 interventions $(67.8 \%)$, three samplings in 40 biopsies $(19.2 \%)$, four samplings in five cases $(2.4 \%)$ and five samplings in one case $(0.5 \%)$ were performed. One attempt was determined especially for risky interventions near great vessels. For the majority of interventions, two samples were sufficient. Three or more bioptic attempts were optimal for large masses with an emphasis on targeting various process parts. The Fisher's exact test proved a statistically non-significant relationship between the number of biop- tic attempts, complication rate $(P=0.46598)$ and histological results accuracy including subtyping $(P=0.34475)$.

False negative results were confirmed in six patients. In four cases, the biopsy revealed the completely necrotic tissue, and in two patients the result was suspected lymphatic affection without any option of classification. However, clinical suspicion of malignant process was still high. The patients were indicated for diagnostic surgical biopsies with wide tumorous tissue excision; the final histological results confirmed germinal cell tumor 3 times, and in one case Hodgkin lymphoma, plasma cell lymphoma and T-cell non-Hodgkin lymphoma. The percutaneous biopsy technique was usual, but the samples were obtained from the non-viable parts of the process (Fig. 4).

In the true negative results, an intensive long-term follow-up was performed. The shortest observation interval was 24 months after the biopsy. A total of 22 patients (22 of 208 biopsies; $10.6 \%$ ) were followed in this group. The most common benign results were chronic inflammatory affection and immunoglubulin G4-related disease (IgG4RD) in 7 cases each (Fig. 5). Granulomatous process was revealed in 4 cases, older hematoma in 2 biopsies and in one case aggressive fibromatosis and accessory splenic tissue were diagnosed. The accuracy of all these diagnoses was confirmed by follow-up CT or PET/CT examinations, where the impairment remained for more than two years without any treatment stationary, decreased in amount or completely dissapeared. 


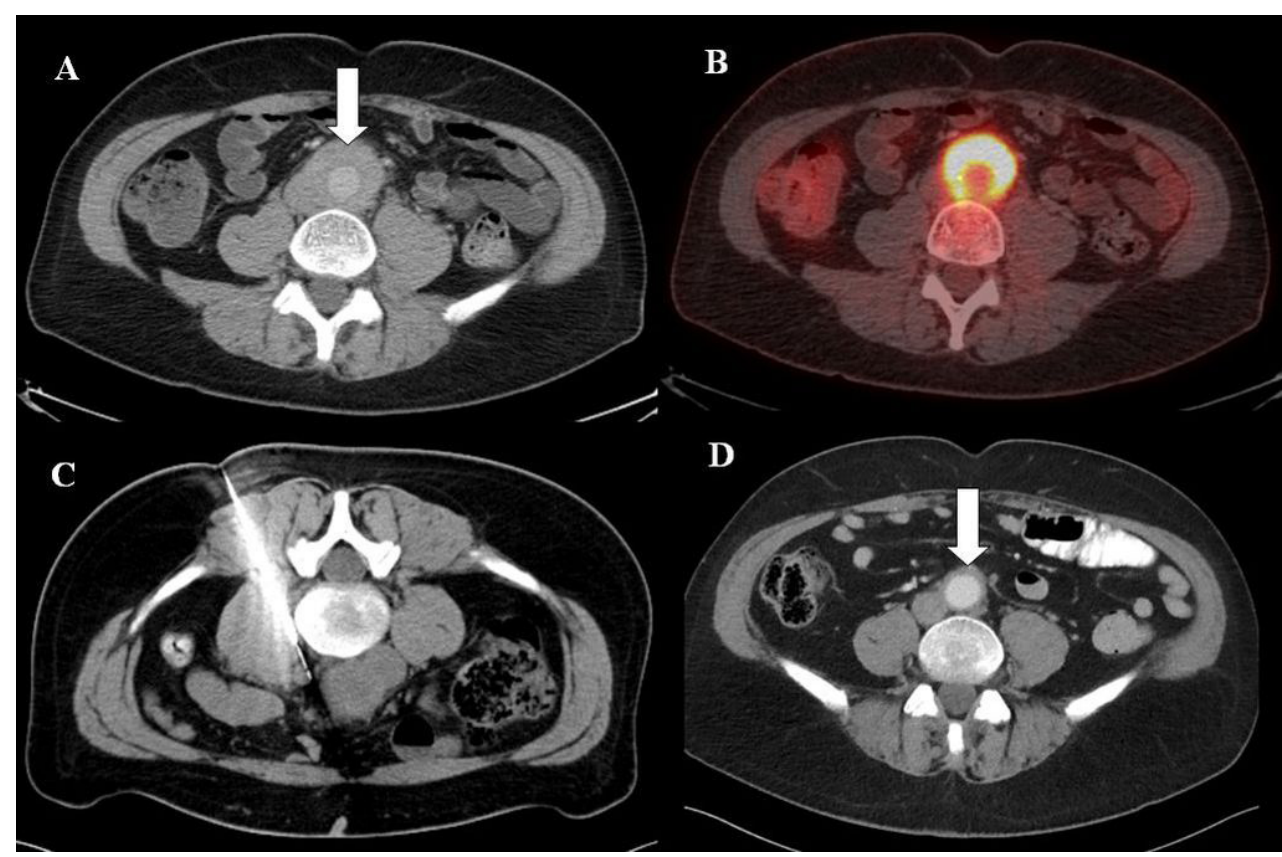

Fig. 5. The true negative result. Vascularized mass around the abdominal aorta on contrast medium enhanced CT (A, arrow) and on FDG enhanced PET/CT (B). The placement of the biopsy needle (C, patient in prone position) with long skin to lesion distance. The histological result was immunoglubulin G4-related disease. The systemic corticosteroid anti-inflammatory treatment was indicated and follow-up CT examination after 6 months verified almost complete regression of the periaortic process.

The complications of the biopsies were observed and correlated with the needle gauge, number of attempts, vascularization, and skin to lesion distance. The statistical data analysis revealed a non-significant relationship between complications incidences and the number of biopsies $(P=0.46598)$ and needle gauges $(P=0.29624)$. In the verified postprocedural hemorrhages, 4 biopsies were performed in hypervascular tumors. The Fisher's exact test proved a statistically significant relationship between the complication incidence and hypervascular process $(P=0.00166)$.

The preprocedural diagnoses were established on the basis of a contrast medium enhanced $\mathrm{CT}$ examination in 151 cases (72.6\%) and on PET/CT examination in 57 cases $(27.4 \%)$. PET/CT was used in all cases with known lymphoma in patients' clinical history due to identification of the most viable tumorous tissue, which is the most suitable for biopsy. Surgical diagnostic excision was indicated in a total of 18 cases $(8.7 \%)$. Skin to lesion distance was variable; from $43 \mathrm{~cm}$ to $178 \mathrm{~cm}$ (median length $108.5 \mathrm{~cm}$ ). The nonparametric Mann-Whitney test proved a statistically significant relationship between the skin to lesion distance and complications $(P=0.218730)$ and hypervascular process character $(P=0.619935)$.

\section{DISCUSSION}

The overall diagnostic accuracy is high; the histological results were true positive or true negative in $97.1 \%$ of cases; subtyping was performed in indicated cases by treating physicians. DLBCL and metastatic disease to retroperitoneal lymphatic nodes were the most common diagnose, with $23.1 \%$ each. Various lymphoma types were revealed in $40.9 \%$ of cases. The true negative results were considered the final diagnoses after long-term follow-up. In $84.6 \%$ of interventions, a thicker biopsy needle of 16 $\mathrm{G}$ was used. In the majority of cases $(89.9 \%)$ at least two bioptic attempts were utilized. The number of bioptic attempts had a statistically non-significant relationship with the complication rate and accuracy of histological results. The skin to lesion distance and hypervascular process had a statistically significant relationship with complications.

The surgical establishment of diagnosis was done with a standard operative procedure under general anesthesia using laparotomy with the most extensive emphasis possible on tumorous tissue resection. Histological examination then proved the origin of the process and potential radicality of treatment. This approach carried all the risks of general anesthesia, resulted in postoperative pain and prolonged recovery time, which was inappropriate for the early beginning of further therapies?

To eliminate these disadvantages, less invasive techniques were developed. To do this, the high diagnostic accuracy had to be preserved. The open surgical approach was replaced by laparoscopic techniques. The substantially high diagnostic accuracy stayed almost unchanged and recovery time became shorter, however general anesthesia was constantly required ${ }^{6,8}$. Percutaneous approach using imaging methods guidance provides the next possibility for obtaining tumorous tissue samples, this time without the necessity of general anesthesia. These procedures are the least invasive and demanding for the patients. Although the first instances of these procedures took 
Table 1. The number of verified diagnoses.

\begin{tabular}{lc}
\hline Diagnosis & Number \\
\hline DLBCL & 48 \\
Metastatic disease & 48 \\
Follicular lymphoma & 21 \\
Hodgkin lymphoma & 11 \\
CLL/SLL & 10 \\
Neuroendocrine tumor & 6 \\
Mixed germinal cell tumor & 4 \\
Dedifferentiated carcinoma & 4 \\
Plasmacytoma & 4 \\
Mantle cell lymphoma & 3 \\
Spindle cell sarcoma & 3 \\
Leiomyosarcoma & 3 \\
Neurinoma & 3 \\
Ganglioneuroma & 2 \\
Marginal zone lymphoma & 1 \\
Extraadrenal myelolipoma & 1 \\
Plasma cell lymphoma & 1 \\
Neurofibroma & 1 \\
T-cell non-Hodgkin lymphoma & 1 \\
Pheochromocytoma & 1 \\
Liposarcoma & 1 \\
Lymphangioma & 1 \\
Myelosarcoma & 1 \\
PNET & 1 \\
No malignant cells verified & 288 \\
$\mathrm{n}$ & \\
\hline &
\end{tabular}

DLBCL, Diffuse Large B-Cell Lymphoma; CLL/SLL, Chronic Lymphocytic Lymphoma/Small Lymphocytic Lymphoma; PNET, Primitive Neuroectodermal Tumor

Table 2. The number of diagnosed metastatic diseases.

\begin{tabular}{lc}
\hline Metastatic diagnosis & Number \\
\hline Renal cell carcinoma & 11 \\
Colorectal carcinoma & 11 \\
Pancreas carcinoma & 5 \\
Breast carcinoma & 4 \\
Urothelial carcinoma & 4 \\
Seminoma & 4 \\
Prostate carcinoma & 3 \\
Cervical carcinoma & 2 \\
NSCLC & 2 \\
Esophageal carcinoma & 1 \\
Duodenal carcinoma & 1 \\
$\mathrm{n}$ & 48 \\
\hline
\end{tabular}

NSCLC, Non Small Cell Lung Carcinoma

place in the 1980's, they have been widely introduced into clinical practise in last decade ${ }^{5,9,10}$.

In literature, there are some articles proving no additional value from biopsies of patients with large resectable masses ${ }^{11}$. These facts can be related to primary tumors. However, the differentiation purely on the basis of imaging examinations without histological verfication cannot be precise. The majority of retroperitoneal affections are systemic diseases. In these cases, systemic targeted treatment is necessary and exact bioptic verification should be performed as precisely, quickly, and with as minimal invasion as possible.

Cytology and aspiration biopsy techniques (Fine Needle Aspiration Biopsy - FNAB) can be sporadically used, but for exact tumorous tissue and especially lymphoma diagnostics including subtyping are not suitable ${ }^{10,12}$. Core needle biopsy (CNB), as the interventional method able to repeatedly obtain satisfactory tissue amounts, is the most preferred minimally invasive procedure and has gradually been accepted as an alternative for deeply located affections, i.e. retroperitoneum ${ }^{13}$. The procedure is quick, well tolerated, inexpensive, safe, and moreover uses only local anesthesia. On the other hand the European Society for Medical Oncology (ESMO) clinical practise guidelines recommend surgical excision biopsies whenever possible, especially in lymphatic nodes affection including suspected malignant lymphomas ${ }^{14,15}$.

Malignant lymphomas are the most common diagnoses of retroperitoneal tumors; our results correspond to this fact. The development of lymphoma treatments has been enormous within recent years. Particular lymphomas however, form heterogenous masses of variable content which can relapse, transform or show decreased sensitivity to treatment. The retroperitoneum is not usually the location of the lymphoma origin, but in cases of relapse or transformation it is frequently affected ${ }^{16,17}$. Therefore, the locations with the largest amount of tissue increase or the most viable part of mass are the most suitable for the biopsy. PET/CT is the preferred examination in patients with known lymphoma in their clinical history ${ }^{18}$. In our group, PET/CT examination was indicated in all cases with suspected known lymphoma relapse or transformation. The correct tissue selection for biopsy plays a crucial role in obtaining an exact histological result and diagnostic accuracy. Nevertheless, the fact is that surgical excision is superior to core needle biopsy in lymphoma transformation diagnostics ${ }^{19}$.

In the literature, there is a reported ambivalent relationship between using a larger needle gauge and improved diagnostic yield. In general, a larger needle size allows for more tumorous tissues to be biopsied and examined. The majority of articles present the use of a 18 $\mathrm{G}$ needle $\mathrm{e}^{5,10,20}$. We used a $18 \mathrm{G}$ needle only in $13 \%$ of procedures; in almost $85 \%$ of interventions we found the $16 \mathrm{G}$ needle suitable. The complication rate was identical and the obtained tissue amount was larger, therefore we consider using wider bioptic instruments to be more appropriate.

In difficult cases, the transvenous approach can be performed, especially in right-sided retroperitoneal affections. The needle track through the inferior vena cava (IVC) or renal vein was published; this article states that biopsies of pancreatic or peripancreatic lesions using needles ranging in size from 18 to $22 \mathrm{G}$ verified no significant increase in complication rate ${ }^{21}$. We used a transcaval approach in a total of five cases; $16 \mathrm{G}$ needle was utilized 3 times and $18 \mathrm{G}$ in two interventions. No serious bleeding complications were verified. 
Various articles can be found regarding retroperitoneal affections and particularly lymphomas using different imaging guidance, approaches and needle gauges ${ }^{10,17,22}$. The first published studies yielded ca. $80-85 \%$ overall accuracy; subtyping rates were not established ${ }^{23,24}$. Recently published studies using improved methods declared both overall and subtyping accuracies exceeding 90-95\% $\left(\right.$ ref. $^{5,17,20}$ ). These results approximate those of surgical excision biopsies. Our results correspond with these published data. Surgical excision biopsies are presented as slightly superior to core needle biopsies ${ }^{19,25}$. On the other hand percutaneous diagnostic procedures are preferred in clinical practise, not only in elderly patients, due to their clear advantages. Gradual improvement of bioptic systems and histological examinations will most likely equalize the accuracy of both approaches within the next few years, assuming that comprehensive studies will provide important experience and will probably lead to integration of core needle biopsies into present guidelines including ESMO.

The limits of our study are the retrospective data analysis, which depends on the hospital information system, absence of a control group, single centre data collection and the smaller number of engaged patients, but this is comparable to published articles.

\section{CONCLUSION}

In conclusion, percutaneous core needle biopsy using CT guidance has high diagnostic accuracy not only in establishing the diagnosis of retroperitoneal disease, but also in its subtyping. Lymphoma development (relapses or transformations to aggressive types) forms a particularly challenging category due to extensive sophisticated histological examination requiring a sufficient amount of representative tissue; the diagnostic accuracy is also high. Core needle biopsies, using at least two samplings of $16 \mathrm{G}$ or a wider calibre needle, the most suitable location, have a low complication rate, are well tolerated by the patients and should be utilized in the retroperitoneal space as an equal procedure to results of surgical biopsies.

Acknowledgements: The authors are grateful to Dr. Olga Cermakova for considerable help with statistical data analysis, to Romana Brezinova for excellent technical support and to Gwyneth Jones for language correction of the manuscipt.

This work was supported by MH CZ - DRO (UHHK, 00179906), by MH CZ NT13531-3/2013 and by SVV 260398/2017.

Author contributions: PD, PH: biopsies performing, manuscript writing, final approval; MB, JK: data analysis; following-up; MH: data analysis, literature search; RD: critical revision, final approval; $\mathrm{MN}$ : histological verification, final approval.

Conflict of interest statement: The authors state that there are no conflicts of interest regarding the publication of this article.

\section{REFERENCES}

1. Tirkes T, Sandrasegaran K, Patel AA, Hollar MA, Tejada JG, Tann M, Akisik FM, Lappas JC. Peritoneal and retroperitoneal anatomy and its relevance for cross-sectional imaging. Radiographics 2012;32(2):43751.

2. Messiou C, Moskovic E, Vanel D, Morosi C, Benchimol R, Strauss D, Miah A, Douis $\mathrm{H}$, van Houdt W, Bonvalot S. Primary retroperitoneal soft tissue sarcoma: Imaging appearances, pitfalls and diagnostic algorithm. Eur J Surg Oncol 2017;43(7):1191-8.

3. Strauss DC, Hayes AJ, Thomas JM. Retroperitoneal tumours: review of management. Ann R Coll Surg Engl 2011;93(4):275-80.

4. Manzella A, Borba-Filho P, D'Ippolito G, Farias M. Abdominal manifestations of lymphoma: spectrum of imaging features. ISRN Radiol 2013;2013:483069.

5. Tomozawa Y, Inaba Y, Yamaura H, Sato Y, Kato M, Kanamoto T, Sakane M. Clinical value of CT-guided needle biopsy for retroperitoneal lesions. Korean J Radiol 2011;12(3):351-7.

6. Sakamoto Y, Karashima R, Ida S, Imamura Y, Iwagami S, Baba Y, Miyamoto Y, Yoshida N, Baba H. Diagnostic laparoscopic biopsy for intraabdominal tumors. Surg Today 2015;45(3):394-6.

7. Morris-Stiff G, Cheang P, Key S, Verghese A, Havard TJ. Does the surgeon still have a role to play in the diagnosis and management of lymphomas? World J Surg Oncol 2008;6:13.

8. Sando M, Terasaki M, Okamoto Y, Suzumura K, Tsuchiya T. The Utility of Diagnostic Laparoscopic Biopsy for Mesenteric and Retroperitoneal Lymph Nodes. Am J Case Rep 2017;18:878-82.

9. Ferrucci JT Jr, Wittenberg J, Mueller PR, Simeone JF, Harbin WP, Kirkpatrick RH, Taft PD. Diagnosis of abdominal malignancy by radiologic fine-needle aspiration biopsy. AJR Am J Roentgenol 1980;134(2):323-30.

10. Shao H, McCarthy C, Wehrenberg-Klee E, Thabet A, Uppot R, Dawson S, Arellano RS. CT-Guided Percutaneous Needle Biopsy of Retroperitoneal and Pelvic Lymphadenopathy: Assessment of Technique, Diagnostic Yield, and Clinical Value. J Vasc Interv Radiol 2018;29(10):1429-36.

11. Chew C, Reid R, O'Dwyer PJ. Value of biopsy in the assessment of a retroperitoneal mass. Surgeon 2006;4(2):79-81.

12. Zhang S, Yu X, Zheng Y, Yang Y, Xie J, Zhou X. Value of fine needle aspiration cell blocks in the diagnosis and classification of lymphoma. Int J Clin Exp Pathol 2014;7(11):7717-25.

13. Skelton E, Jewison A, Okpaluba C, Sallomi J, Lowe J, Ramesar K, Grace $\mathrm{R}$, Howlett DC. Image-guided core needle biopsy in the diagnosis of malignant lymphoma. Eur J Surg Oncol 2015;41:852-8.

14. Ladetto M, Buske C, Hutchings M, Dreyling M, Gaidano G, Le Gouill S, Luminari S, Pott C, Zamò A, Zucca E; \& the ESMO Lymphoma Consensus Conference Panel Members. ESMO consensus conference on malignant lymphoma: general perspectives and recommendations for prognostic tools in mature B-cell lymphomas and chronic lymphocytic leukaemia. Ann Oncol 2018;29:525.

15. Buske C, Hutchings M, Ladetto M, Goede V, Mey U, Soubeyran P, Spina M, Stauder R, Trnený M, Wedding U, Fields P; ESMO Lymphoma Consensus Conference Panel Members. ESMO Consensus Conference on malignant lymphoma: general perspectives and recommendations for the clinical management of the elderly patient with malignant lymphoma. Ann Oncol 2018;29:544-562.

16. Acar T, Harman M, Guneyli S, Gemici K, Efe D, Guler I, Yildiz M. Crosssectional Imaging Features of Primary Retroperitoneal Tumors and Their Subsequent Treatment. J Clin Imaging Sci 2015;5:24.

17. Schiavon LHO, Tyng CJ, Travesso DJ, Rocha RD, Schiavon ACSA, Bitencourt AGV. Computed tomography-guided percutaneous biopsy of abdominal lesions: indications, techniques, results, and complications. Radiol Bras 2018;51(3):141-6.

18. Tamayo P, Martín A, Díaz L, Cabrero M, García R, García-Talavera P, Caballero D. 18F-FDG PET/CT in the clinical management of patients with lymphoma. Rev Esp Med Nucl Imagen Mol 2017;36(5):312-21.

19. Johl A, Lengfelder E, Hiddemann W, Klapper W; German Low-grade Lymphoma Study Group (GLSG). Core needle biopsies and surgical excision biopsies in the diagnosis of lymphoma-experience at the Lymph Node Registry Kiel. Ann Hematol 2016;95(8):1281-6.

20. Stattaus J, Kalkmann J, Kuehl H, Metz KA, Nowrousian MR, Forsting M, Ladd SC. Diagnostic yield of computed tomography-guided coaxial core biopsy of undetermined masses in the free retroperito- 
neal space: single-center experience. Cardiovasc Intervent Radiol 2008;31(5):919-25.

21. Sofocleous CT, Schubert J, Brown KT, Brody LA, Covey AM Getrajdman GI. CT-guided transvenous or transcaval needle biopsy of pancreatic and peripancreatic lesions. J Vasc Interv Radiol 2004;15(10):1099-104.

22. Özel D, Aydın T. A clinical compilation of lymph node pathologies comparing the diagnostic performance of biopsy methods. Ultrasound 2019;22(1):59-64. doi: 10.1007/s40477-018-0321-6.

23. Knelson M, Haaga J, Lazarus H, Ghosh C, Abdul-Karim F, Sorenson
K. Computed tomography-guided retroperitoneal biopsies. J Clin Oncol 1989;7(8):1169-73.

24. Fisher AJ, Paulson EK, Sheafor DH, Simmons CM, Nelson RC. Small lymph nodes of the abdomen, pelvis, and retroperitoneum: usefulness of sonographically guided biopsy. Radiology 1997;205(1):18590.

25. Daly SC, Klairmont M, Arslan B, Vigneswaran Y, Roggin KF, Ujiki MB, Denham W, Millikan KW, Luu MB, Deziel DJ, Myers JA. Laparoscopy has a superior diagnostic yield than percutaneous image-guided biopsy for suspected intra-abdominal lymphoma. Surg Endosc 2015;29:2496-9. 